\title{
Assessment of The Association of OCT3/4 With GLUT1 And CD105 In Oral Squamous Cell Carcinoma Using Dual Immunohistochemistry
}

\section{Samira Derakhshan}

Tehran University of Medical Sciences

Nazanin Mahdavi

Tehran University of Medical Sciences

Neda Kardouni Khoozestani

Tehran University of Medical Sciences

Bita Nasr Esfahani

Tehran University of Medical Sciences

\section{Forouzan Heidarian}

Tehran University of Medical Sciences

Ali Abolrahmani ( $\nabla$ a-abdolrahmani@alumnus.tums.ac.ir)

Tehran University of Medical Sciences

\section{Sedigheh Rahrotaban}

Tehran University of Medical Sciences

\section{Research Article}

Keywords: Angiogenesis, Cancer stem cell, Dual immunohistochemistry, GLUT1, OCT3/4, Squamous cell carcinoma

Posted Date: January 7th, 2022

DOI: https://doi.org/10.21203/rs.3.rs-1204785/v1

License: (c) (1) This work is licensed under a Creative Commons Attribution 4.0 International License. Read Full License 


\section{Abstract}

Background: Oral squamous cell carcinoma (OSCC) is the most common cancer type affecting the oral and maxillofacial region. This study aimed to investigate the role of cancer stem cells (CSCs) in angiogenesis and hypoxic response in OSCC.

Methods: This retrospective observational study evaluated 56 cases of OSCC using dual immunohistochemistry. Octamer-binding transcription factor 3/4 (OCT3/4) marker was used for evaluation of CSC activity. Glucose transporter 1 (GLUT1) marker was used to evaluate the hypoxic response and angiogenesis, while endoglin (CD105) was used to evaluate the late stage of angiogenesis and blood vessel formation.

Results: Overexpression of both OCT3/4 and GLUT1 was noted in early stage of hypoxic response and angiogenesis in OSCC, indicating the important role of CSCs in this phase. However, despite higher expression of CD105, the expression of CSC markers was not significant in the late stage of tumor progression and angiogenesis.

Conclusions: CSCs could play important roles in initial stages of tumor progression and angiogenesis. Further studies are required to discover other biomarkers, their roles, and associated pathways of CSCs in OSCC.

\section{Introduction}

Oral squamous cell carcinoma (OSCC) accounts for approximately $90 \%$ of all oral malignancies. In addition to its high prevalence, OSCC has a poor five-year survival rate of about $50 \% .(1,2)$ Despite the advancements made in surgical techniques and adjuvant therapeutic modalities, long-term survival rate of OSCC patients has only shown a modest improvement.(3) Therefore, aside from the conventional TNM staging and histopathological differentiation, there is an evident need for more accurate and reliable prognostic markers to identify high-risk patients.

Since the identification of cancer stem cells (CSCs) by Bonnet in 1997,(4) their role in hierarchical organization of cancer cells has received wide attention. In this process, angiogenesis and cellular heterogeneity are maintained by only a small group of cells which have the ability to self-renew and differentiate. These cells may actually be responsible for resistance to radiation and chemotherapy.(5) Thus, CSCs are recognized as one of the most important factors responsible for failure of conventional cancer therapy. This statement led to the development of a new paradigm in treatment of malignant lesions.(6) Octamer-binding transcription factor 3/4 (OCT3/4), also known as POU5F1 and Oct4, is a master factor for CSC functions such as self-renewal and differentiation, and may play a critical role in developing resistance to conventional cancer therapy.(7) Alterations in tumor micro-environment such as hypoxia have a significant effect on tumor progression and CSC functions through modification of metabolic pathways and production of hypoxia-inducible factor-1a. $(6,8)$ Uncontrolled oncogene-induced proliferation, in absence of efficient blood supply, results in hypoxia. The tumors react to hypoxia by 
inducing angiogenesis and metabolic remodeling to enhance their progression.(9) Therefore, angiogenesis is fundamental for progression, invasion, and metastasis of tumors. Many signaling pathways play essential roles in angiogenesis; therefore, angiogenesis can be detected or inhibited through such pathways.(10) At preliminary stages, glucose transporter-1 (GLUT1) expression increases in response to hypoxia-inducible factor-1a.(11) Also, owing to the role of GLUT1 in tumor progression and metabolism, overexpression of GLUT1 could predict a more aggressive behavior and a worse clinical outcome.(12) Endoglin (CD105) is part of the transforming growth factor beta receptor complex, which is up-regulated in proliferating endothelial cells.(13) Lack of sufficient information about the correlation of the expression of CSC markers and angiogenesis in head and neck carcinomas specially the oral squamous cell carcinoma (OSCC) encouraged us to further scrutinize this correlation in OSCC. In order to investigate the role of CSCs in tumor response to hypoxia, we assessed the GLUT-1 and CD105 as markers of hypoxic response and angiogenesis in this study.

\section{Materials And Methods}

\section{Samples}

A total of 56 formalin-fixed, paraffin embedded tissue specimens diagnosed with OSCC were selected from the archives of the Oral and Maxillofacial Pathology Department of Dental School of Tehran University of Medical Sciences. The blocks were inspected again to confirm the diagnosis, and also for the assessment of histological tumor grade. Specimens with optimal tissue adequacy were selected. Histopathological tumor grading was performed according to the 2017 World Health Organization classification of head and neck tumors as: low (I), moderate (II) or high (III) grade.(14) Demographic information of patients and clinico-histopathological features of the tumors were collected from the records. This cross-sectional study was carried out in two phases and supported by Tehran University of Medical Sciences, School of Dentistry grant \#98-01-69-41495 and \#98-02-69-42939, and was approved by the ethics in research committee (registration codes IR.TUMS.DENTISTRY.REC.1398.010 and IR.TUMS.DENTISTRY.REC.1399.164).

\section{Staining}

The specimens were sectioned into 3- $\mu$ m thick slices. Immunohistochemical (IHC) staining was performed using a dual staining kit which is used for simultaneous evaluation of two or more antigens. We used the master dual staining kit (MAD-001882QK, Master Diagnostica, Granada, Spain) according to the provided instructions to evaluate the expression of GLUT1, CD105, and OCT3/4 markers. Two sections of each block were stained. The first section was stained with CD105 and OCT3/4, and the second section was stained with GLUT1 and OCT3/4. Dual staining requires two types of antibodies from two different animal sources; thus, we used goat polyclonal antibody to detect OCT3/4 and rat monoclonal antibodies to detect CD105 and GLUT1 (Table 1). 
Table 1

Antibodies information used for immunohistochemistry examination.

\begin{tabular}{|lllllll|}
\hline Antibody & $\begin{array}{l}\text { Type of } \\
\text { Antibody }\end{array}$ & Host Animal & Brand & Catalog Number & Clone & Dilution \\
\hline OCT 3/4 & Polyclonal & Rabbit & ABCAM & ab19857 & - & $1: 100$ \\
\hline CD105 & Monoclonal & Mouse & Biotechne & MAB1320 & 209701 & $1: 100$ \\
\hline GLUT1 & Monoclonal & Mouse & Santa Cruz & sc-377228 & (A-4) & $1: 50$ \\
\hline
\end{tabular}

\section{Evaluation}

Each stained block was separately scored by two senior pathologists. Brown staining of the nuclei was regarded as a positive result for OCT3/4. The intensity and percentage of staining were both evaluated for 0 CT $3 / 4$ scoring. The intensity of staining was rated from 0 to $3(0=$ no staining, $1=$ mild staining, $2=$ moderate staining, $3=$ sever staining). Distribution of staining was rated from 0 to 3 ( $0=$ no cell is stained, $1=$ less than $10 \%$ of cells are stained, $2=10$ to $50 \%$ of cells are stained and $3=$ more than $50 \%$ of cells are stained). The total score (0-9) was calculated by multiplying the intensity score and distribution score, and scores 0-2 indicated low, 3-4 indicated moderate, and 6-9 indicated severe expression.(15)

Red staining of cell cytoplasm was regarded as a positive result for angiogenesis (CD105). To evaluate angiogenesis, the first step was to determine the highly stained fields (neovascularization hot spots) at low magnification. The exact scoring was subsequently performed at high magnification by evaluating four areas identified with the highest staining intensity.(16) Only CD105 positive expression was used to describe a cell merely stained with CD105 antibody and had endothelial differentiation without CSC origin. The surrounding connective tissue endothelial cells were used as the internal positive control.

For evaluation of GLUT1 immunoreactivity, both membranous and cytoplasmic staining were considered as a positive result. The percentage of positively stained tumoral cells was graded as score 0 (no positive cell), 1 (1-10\% positive tumoral cells), 2 (11-50\% positive tumoral cells), 3 (50-80\% positive tumoral cells), and 4 (81-100\% positive tumoral cells).(17) Finally, scores 0,1 and 2 were regarded as low expression and scores 3 and 4 were regarded as high expression of GLUT1.

In concurrent staining of cells for angiogenesis markers and OCT3/4 antibody, red cytoplasmic and brown nuclear staining of the same cell was used as the criterion for co-expression and regarded as a positive result. The co-expression score was also determined. For this purpose, two fields that had the highest and lowest concentration of cells stained by both markers at low magnification were first selected. The co-expression scores were determined by comparing the cell count in the afore-mentioned two fields at high magnification. The obtained scores were then compared among the samples.

\section{Data analysis}


The data were analyzed using SPSS 25 (IBM SPSS Statistics for Windows, Armonk, NY, USA) by the nonparametric Mann-Whitney $U$ and Kruskal-Wallis tests. The Kruskal-Wallis test was used to compare the expression of CD105, OCT3/4, and GLUT1 separately in tumoral cells and co-expression of these markers in different OSCC grades by calculating the mean rank. In using the non-parametric Mann-Whitney $\mathrm{U}$ test, grades 1 and 2 were considered as one group and the expression in this merged group was compared with that in grade 3 group. $P$ values less than 0.05 were considered statistically significant.

\section{Results}

Positive immunoreactivity of tumoral cells in dual staining method is shown in Figure 1. Tumor samples belonged to 21 female (37.5\%) and 35 male (62.5\%) patients. The histopathological grade was grade 1 in 27 patients, grade 2 in 15 cases, and grade 3 or poorly differentiated in 14 cases (Table 2). Tumor grade only had a significant correlation with CD105 expression $(p=0.039)$. CD105 expression increased in higher tumor grades (Table 3). After merging of grades 1 and 2 and comparison with grade 3 , there was no significant correlation between CD105, OCT3/4 or their co-expression with histological grade (Table 4). Six samples were excluded in step 2 for GLUT1 evaluation because of technical errors. The final score of GLUT1 revealed that all the samples with histological grade 1 had low expression of GLUT1 (Table 5). But in co-expression of two antibodies together, $91.7 \%$ of the cases with histological grade 3 demonstrated high expression of antibodies, which was significant $(p<0.05$, Table 6$)$.

Table 2

Tumor grade and gender distribution.

\begin{tabular}{|c|c|c|c|c|c|}
\hline & & & \multicolumn{2}{|l|}{ gender } & \multirow[t]{2}{*}{ Total } \\
\hline & & & Female & Male & \\
\hline \multicolumn{6}{|c|}{ Grade } \\
\hline & \multirow[t]{2}{*}{1} & Count & 9 & 18 & 27 \\
\hline & & $\%$ within grade & $33.3 \%$ & $66.7 \%$ & $100.0 \%$ \\
\hline & \multirow[t]{2}{*}{2} & Count & 8 & 7 & 15 \\
\hline & & $\%$ within grade & $53.3 \%$ & $46.7 \%$ & $100.0 \%$ \\
\hline & \multirow[t]{2}{*}{3} & Count & 4 & 10 & 14 \\
\hline & & $\%$ within grade & $28.6 \%$ & $71.4 \%$ & $100.0 \%$ \\
\hline \multirow[t]{2}{*}{ Total } & & Count & 21 & 35 & 56 \\
\hline & & $\%$ within grade & $37.5 \%$ & $62.5 \%$ & $100.0 \%$ \\
\hline
\end{tabular}


Table 3

Correlation of antibodies expression with grade.

\begin{tabular}{|c|c|c|c|c|c|}
\hline & & grade & CD105 & Co-expression* & ОСТЗ/4 \\
\hline \multicolumn{6}{|l|}{ grade } \\
\hline & Pearson Correlation & 1 & .277 & 0.222 & 0.098 \\
\hline & Sig. (2-tailed) & - & 0.039 & 0.100 & 0.472 \\
\hline & $\mathrm{N}$ & 56 & 56 & 56 & 56 \\
\hline \multicolumn{6}{|l|}{ CD105 } \\
\hline & Pearson Correlation & .277 & 1 & .734 & .526 \\
\hline & Sig. (2-tailed) & 0.039 & - & 0.000 & 0.000 \\
\hline & $\mathrm{N}$ & 56 & 56 & 56 & 56 \\
\hline \multicolumn{6}{|c|}{ Co expression* } \\
\hline & Pearson Correlation & 0.222 & .734 & 1 & .649 \\
\hline & Sig. (2-tailed) & 0.100 & 0.000 & - & 0.000 \\
\hline & $\mathrm{N}$ & 56 & 56 & 56 & 56 \\
\hline \multicolumn{6}{|c|}{ ОСТ3/4 } \\
\hline & Pearson Correlation & 0.098 & .526 & .649 & 1 \\
\hline & Sig. (2-tailed) & 0.472 & 0.000 & 0.000 & - \\
\hline & $\mathrm{N}$ & 56 & 56 & 56 & 56 \\
\hline
\end{tabular}

Table 4

Correlation of antibodies expression with grade in the merging groups.

\begin{tabular}{|lllll|}
\hline & CD105 & Co-expression** & OCT3/4 & only CD105*** \\
\hline Kruskal-Wallis H & 3.906 & 3.319 & 0.682 & 1.588 \\
\hline $\mathrm{df}$ & 2 & 2 & 2 & 2 \\
\hline Asymp. Sig. & 0.142 & 0.190 & 0.711 & 0.452 \\
\hline $\begin{array}{l}\text { * expresion of CD105 marker with/ without expression of CD105. } \\
\text { OCT }\end{array}$ & OCo-expression of both CD105 and \\
\hline
\end{tabular}


Table 5

Correlation of GLUT1 score with grade.

\begin{tabular}{|c|c|c|c|c|c|}
\hline & & & \multicolumn{2}{|c|}{ GLUT1 score } & \multirow[t]{2}{*}{ Total } \\
\hline & & & 1 & 2 & \\
\hline \multicolumn{6}{|c|}{ Grade } \\
\hline & \multirow[t]{2}{*}{1} & Count & 22 & 0 & 22 \\
\hline & & $\%$ within grade & $100.0 \%$ & $0.0 \%$ & $100.0 \%$ \\
\hline & \multirow[t]{2}{*}{2} & Count & 11 & 5 & 16 \\
\hline & & $\%$ within grade & $68.8 \%$ & $31.3 \%$ & $100.0 \%$ \\
\hline & \multirow[t]{2}{*}{3} & Count & 7 & 5 & 12 \\
\hline & & $\%$ within grade & $58.3 \%$ & $41.7 \%$ & $100.0 \%$ \\
\hline \multirow[t]{2}{*}{ Total } & & Count & 40 & 10 & 50 \\
\hline & & $\%$ within grade & $37.5 \%$ & $62.5 \%$ & $100.0 \%$ \\
\hline
\end{tabular}

Table 6

Correlation of co-expression of GLUT1 and OCT3/4 with grade.

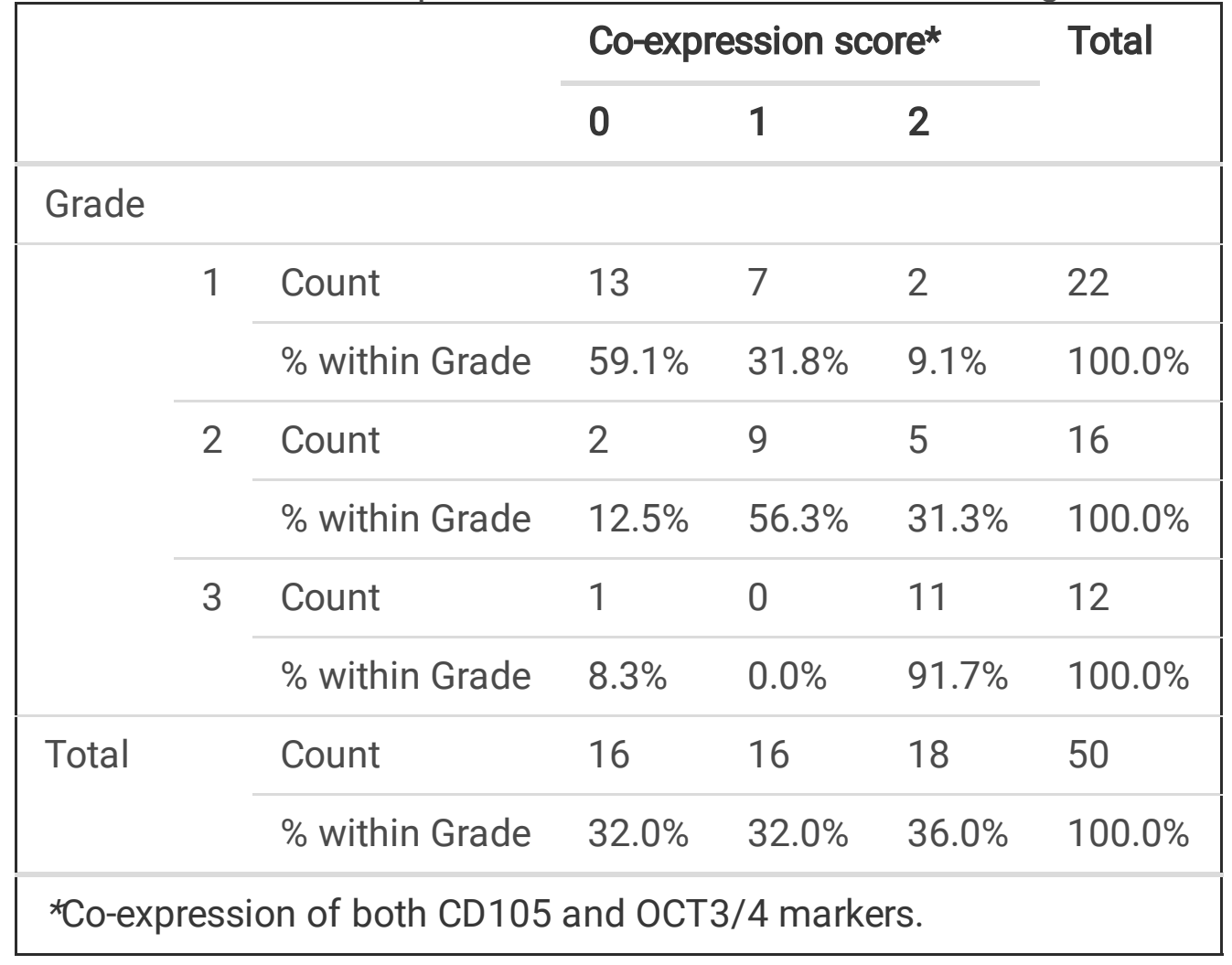

As shown in Table 7, the histological grade was significantly correlated with count, intensity, and final scores of OCT3/4, count and final scores of GLUT1, and also co-expression of these two antibodies ( $p<$ $0.05)$. 
Table 7

Correlation of antibodies expression with grade.

\begin{tabular}{|c|c|c|c|c|c|c|}
\hline & $\begin{array}{l}\text { ОСТ3/4 } \\
\text { percentage* }\end{array}$ & $\begin{array}{l}\text { OCT3/4 } \\
\text { intensity score }\end{array}$ & $\begin{array}{l}\text { OCT3/4 } \\
\text { final score }\end{array}$ & $\begin{array}{l}\text { GLUT1 } \\
\text { percentage }\end{array}$ & $\begin{array}{l}\text { GLUT1 } \\
\text { final } \\
\text { score }\end{array}$ & $\begin{array}{l}\text { Co- } \\
\text { expression } \\
\text { score }^{\star \star *}\end{array}$ \\
\hline $\begin{array}{l}\text { Asymp. } \\
\text { Sig. } \\
\text { with } \\
\text { Grade }\end{array}$ & .000 & .011 & .000 & .000 & .006 & .000 \\
\hline
\end{tabular}

\section{Discussion}

Recent studies support the correlation of CSCs and angiogenesis in different carcinomas. The population of CSCs increases in hypoxia, which results in rapid tumor growth and metastasis.(18) Also, CSCs can produce higher levels of vascular endothelial growth factor (one of the most important angiogenesis inducing factors)(19) than other malignant tumoral cells.(20) Thus, combination of anti-angiogenetic drugs and anti-CSC drugs together with conventional cancer therapy is highly promising.(21)

Studies on OSCC have shown the fundamental role of GLUT1 and CD105 in hypoxic response and correlation of their expression with aggressive behavior and worse clinical outcomes.(12, 22, 23) Also, studies on OSCC demonstrated OCT3/4 as a potential prognostic marker correlated with poor overall survival rate, and suggested further studies on its expression in conjunction with other markers.(24)

Considering the overexpression of GLUT1 in pre-cancerous lesions, it may have a more prominent role in early stages of malignant transformation;(25) whereas, CD105 represents the formed vessels and is associated with late stages of angiogenesis and hypoxic response. We used these two markers in this study to elucidate the role of CSCs in early and late stages of hypoxic response.

Our results suggest that in early stage of hypoxic situation in OSCC, CSCs may be responsible for hypoxic response due to overexpression of both OCT3/4 and GLUT1. But in late stage of tumor progression and angiogenesis with higher expression of CD105 which leads to angiogenesis and blood vessel formation, the expression of CSC markers is not significant, which means a reduction in the role of CSCs in angiogenesis in the late stage of tumor progression.

Also, the current results indicated that overexpression of both OCT3/4 and GLUT1 markers was correlated with poor prognosis in OSCC. Thus, CSCs may be associated with poorer prognosis via GLUT1 expression in hypoxic conditions. This finding was in accordance with recent studies that showed tumor hypoxia was associated with a poorer prognosis in head and neck cancers. $(26,27)$ 
Recently, CSCs have been identified as potentially initiating cells of tumor angiogenesis and neovascularization. Evidence strongly suggests that association of CSCs with tumor angiogenesis may be mediated through the induction of vascular endothelial growth factor production.(28) Also, CSCs can organize a pseudo-vascular network in some malignant tumors.(28) It might be the result of their differentiation into a borderline transitional cell type between an endothelial cell and a tumor cell among tumoral cells. Further studies are necessary to evaluate these processes. CSCs are diverse and have various markers. They vary in different grades and stages of a tumor, in different tumors, and also in different patients with the same type of tumor. We evaluated only one CSC marker (OCT3/4), which was a limitation of the present study.

In recent years, utilization of dual staining methods has considerably increased. Two or more antigens can be evaluated simultaneously by using dual staining. This method can reveal the correlation of different antigens, and may also increase the chance of cross-reactions. Various procedures have been suggested to reduce these cross-reactions such as using antibodies from different species.(29)

To our knowledge, it is the first study to evaluate the expression of GLUT1 and CD105 hypoxic marker as markers of angiogenesis in association with one CSC marker in OSCC, via dual staining immunohistochemistry. We showed that CSCs could play an important role in initial stages of tumor progression and angiogenesis. Further studies are required to discover the roles, biomarkers and associated pathways of CSCs in OSCC.

\section{Abbreviations}

OSCC: Oral squamous cell carcinoma

CSCs: Cancer stem cells

IHC: Immunohistochemistry.

ОСТ3/4: Octamer-binding transcription factor $3 / 4$

GLUT1: Glucose transporter 1

CD105: Endoglin

\section{Declarations}

\section{Ethics approval}

All methods were carried out in accordance with relevant guidelines and regulations. All experimental protocols were approved by the Biomedical Research Ethics Committee of Tehran University of Medical Sciences via registration codes IR.TUMS.DENTISTRY.REC.1398.010 and IR.TUMS.DENTISTRY.REC.1399.164. 


\section{Consent to participate}

Informed consent was obtained from all subjects and/or their legal guardian(s).

\section{Consent for publication}

Not applicable.

\section{Availability of data and materials}

The datasets used and/or analyzed during the current study available from the corresponding author on reasonable request.

\section{Competing interests}

The authors have no conflict of interests to declare.

\section{Funding}

This study was supported by a grant from Tehran University of Medical Sciences, School of Dentistry (grants \#98-01-69-41495 and \#98-02-69-42939).

\section{Authors' contributions}

SD and NM conceptualized research and established methodology, SD and NK conducted examination and evaluated the $\mathrm{IHC}$ results $\mathrm{FH}$ and $\mathrm{BN}$ analyzed and interpreted the result. $\mathrm{AA}$, NK and SD were the major contributor in writing the manuscript. BN and SR wrote review and edited the manuscript. All authors read and approved the final manuscript.

\section{Acknowledgement}

The authors would like to thank Dr. Mohammad Javad Kharrazi Fard of Dental Research Center, School of Dentistry, Tehran University of Medical Sciences, for his assistance in data analysis.

\section{References}

1. Feller L, Lemmer J. Oral Squamous Cell Carcinoma: Epidemiology, Clinical Presentation and Treatment. Journal of Cancer Therapy. 2012;03.

2. Vigneswaran N, Williams MD. Epidemiologic trends in head and neck cancer and aids in diagnosis. Oral Maxillofac Surg Clin North Am. 2014;26:123-41.

3. Johnson DE, Burtness B, Leemans CR, Lui VWY, Bauman JE, Grandis JR. Head and neck squamous cell carcinoma. Nature Reviews Disease Primers. 2020;6:92.

4. Bonnet D, Dick JE. Human acute myeloid leukemia is organized as a hierarchy that originates from a primitive hematopoietic cell. Nat Med. 1997;3:730-7. 
5. Visvader JE, Lindeman GJ. Cancer stem cells: current status and evolving complexities. Cell Stem Cell. 2012;10:717-28.

6. Menendez JA, Joven J, Cufi S, Corominas-Faja B, Oliveras-Ferraros C, Cuyas E, et al. The Warburg effect version 2.0: metabolic reprogramming of cancer stem cells. Cell Cycle. 2013;12:1166-79.

7. Mohiuddin IS, Wei S-J, Kang MH. Role of OCT4 in cancer stem-like cells and chemotherapy resistance. Biochimica et Biophysica Acta (BBA) - Molecular Basis of Disease. 2019:165432.

8. Semenza GL. Oxygen sensing, hypoxia-inducible factors, and disease pathophysiology. Annu Rev Pathol. 2014;9:47-71.

9. Eales KL, Hollinshead KER, Tennant DA. Hypoxia and metabolic adaptation of cancer cells. Oncogenesis. 2016;5:e190-e.

10. Dimova I, Popivanov G, Djonov V. Angiogenesis in cancer - general pathways and their therapeutic implications. J buon. 2014;19:15-21.

11. Wu XH, Chen SP, Mao JY, Ji XX, Yao HT, Zhou SH. Expression and significance of hypoxia-inducible factor-1a and glucose transporter-1 in laryngeal carcinoma. Oncol Lett. 2013;5:261-6.

12. Li C-X, Sun J-L, Gong Z-C, Lin Z-Q, Liu H. Prognostic value of GLUT-1 expression in oral squamous cell carcinoma: A prisma-compliant meta-analysis. Medicine (Baltimore). 2016;95:e5324-e.

13. Nassiri F, Cusimano MD, Scheithauer BW, Rotondo F, Fazio A, Yousef GM, et al. Endoglin (CD105): a review of its role in angiogenesis and tumor diagnosis, progression and therapy. Anticancer Res. 2011;31:2283-90.

14. El-Naggar AK, Chan JK, Grandis JR. WHO classification of head and neck tumours2017.

15. Naini F, Kamyab K, Mahdavi N, Derakhshan S. OCT-4 Is a Good Predictive Biomarker for Local Recurrence in Head and Neck Basal Cell Carcinoma. Journal of Islamic Dental Association of IRAN. 2017;29:58-63.

16. Ascani G, Balercia P, Messi M, Lupi L, Goteri G, Filosa A, et al. Angiogenesis in oral squamous cell carcinoma. Acta Otorhinolaryngol Ital. 2005;25:13-7.

17. Roh JL, Cho KJ, Kwon GY, Ryu CH, Chang HW, Choi SH, et al. The prognostic value of hypoxia markers in T2-staged oral tongue cancer. Oral Oncol. 2009;45:63-8.

18. Conley SJ, Gheordunescu E, Kakarala P, Newman B, Korkaya H, Heath AN, et al. Antiangiogenic agents increase breast cancer stem cells via the generation of tumor hypoxia. Proc Natl Acad Sci U S A. 2012;109:2784-9.

19. Al-Husein B, Abdalla M, Trepte M, Deremer DL, Somanath PR. Antiangiogenic therapy for cancer: an update. Pharmacotherapy. 2012;32:1095-111.

20. Eyler CE, Rich JN. Survival of the fittest: cancer stem cells in therapeutic resistance and angiogenesis. J Clin Oncol. 2008;26:2839-45.

21. Markowska A, Sajdak S, Markowska J, Huczyński A. Angiogenesis and cancer stem cells: New perspectives on therapy of ovarian cancer. European Journal of Medicinal Chemistry. 2017;142:8794. 
22. Lin W, Yin C-Y, Yu Q, Zhou S-H, Chai L, Fan J, et al. Expression of glucose transporter-1, hypoxia inducible factor-1 $\mathrm{a}$ and beclin-1 in head and neck cancer and their implication. Int $\mathrm{J}$ Clin Exp Pathol. 2018;11:3708-17.

23. Sakurai T, Okumura H, Matsumoto M, Uchikado Y, Owaki T, Kita Y, et al. Endoglin (CD105) is a useful marker for evaluating microvessel density and predicting prognosis in esophageal squamous cell carcinoma. Anticancer Res. 2014;34:3431-8.

24. Gliagias V, Wotman M, Herman SW, Costantino P, Kraus D, Tham T. Investigating the role of octamer binding transcription Factor-4 (Oct-4) in oral cavity squamous cell carcinoma: A systematic review and meta-analysis. Am J Otolaryngol. 2019;40:282-8.

25. Pereira KM, Feitosa SG, Lima AT, Luna EC, Cavalcante RB, de Lima KC, et al. Immunohistochemical Evaluation of Glucose Transporter Type 1 in Epithelial Dysplasia and Oral Squamous Cell Carcinoma. Asian Pac J Cancer Prev. 2016;17:147-51.

26. Kaanders JH, Wijffels KI, Marres HA, Ljungkvist AS, Pop LA, van den Hoogen FJ, et al. Pimonidazole binding and tumor vascularity predict for treatment outcome in head and neck cancer. Cancer Res. 2002;62:7066-74.

27. Jonathan RA, Wijffels KI, Peeters W, de Wilde PC, Marres HA, Merkx MA, et al. The prognostic value of endogenous hypoxia-related markers for head and neck squamous cell carcinomas treated with ARCON. Radiother Oncol. 2006;79:288-97.

28. Ping YF, Bian XW. Cancer stem cells switch on tumor neovascularization. Curr Mol Med. 2011;11:6975.

29. Waiser J, Schwaar S, Bohler T, Rudolph B, Dell K, Budde K, et al. Immunohistochemical doublestaining of renal allograft tissue: critical assessment of three different protocols. Virchows Arch. 2002;440:648-54.

\section{Figures}

\section{Figure 1}

Dual immunostaining results; Positive brown nuclear immunostaining for OCT3/4 in tumoral cells $(\times 100$ magnification). (A) Positive brown nuclear immunostaining for OCT3/4 and red cytoplasmic immunostaining for CD105 in tumoral cells and some blood vessel structures ( $\times 400$ magnification). (B) Positive red cytoplasmic and membranous immunostaining for GLUT1 in tumoral cells and some blood vessels ( $\times 100$ magnification). (C) Positive red immunostaining of GLUT1 and positive brown immunostaining for OCT3/4 in tumoral cells ( $\times 100$ magnification). (D) 